\title{
On Supervisory Control of Concurrent Discrete-Event Systems
}

\author{
Yosef Willner* Michael Heymann ${ }^{\dagger}$
}

March 27, 2002

\begin{abstract}
When a discrete-event system $P$ consists of several subsystems $P_{1}, \ldots, P_{n}$ that operate concurrently, a natural approach to the supervisory control problem is to synthesize a 'local' controller $S_{i}$ for each subsystem $P_{i}$ and operate the individually controlled subsystems $S_{i} / P_{i}$ concurrently. Such an approach is called concurrent supervisory control and is closely related to decentralized supervisory control as studied in [4] and [5]. In the present paper simple and easily computable conditions are developed, that guarantee when concurrent supervisory control can achieve the optimal behavior achievable by a global supervisor. To achieve the optimal behavior, two specific concurrent control strategies are introduced.
\end{abstract}

Key words: discrete event systems, supervisory control, concurrency, decentralization, separability.

\section{Introduction}

In recent years a control theory for a general class of Discrete Event Systems (DES) has been proposed by Ramadge and Wonham [1-3]. In this theory, the DES is modelled as a tuple

$$
P=\left(Q, \Sigma, \delta, q_{0}\right)
$$

${ }^{*}$ Department of Electrical Engineering, Technion - Israel Institute of Technology, Haifa 32000, Israel.

${ }^{\dagger}$ Department of Computer Science, Technion - Israel Institute of Technology, Haifa 32000, Israel. Completed in part while the author was on leave as a NRC-Senior Research Associate at NASA-Ames Research Center, Moffett Field, CA 94035. 
where $Q$ is a set of states, $\Sigma$ is a set of symbols called the transition alphabet, $\delta: \Sigma \times Q \rightarrow Q$ is a (partial) function called the state transition function, and $q_{0} \in Q$ is the initial state. ${ }^{1}$

Let $\Sigma^{*}$ denote the set of all strings over $\Sigma$, including the empty string $\epsilon$. A subset $\mathcal{L} \subset \Sigma^{*}$ is called a language over $\Sigma$. A string $u \in \Sigma^{*}$ is a prefix of $v \in \Sigma^{*}$, denoted $u<v$, if for some string $w \in \Sigma^{*}, v=u w$. The closure of $\mathcal{L} \subset \Sigma^{*}$, denoted $\overline{\mathcal{L}}$, is the set of all prefixes of strings in $\mathcal{L}$. $\mathcal{L}$ is closed if $\overline{\mathcal{L}}=\mathcal{L}$.

For a DES $P$, the transition function $\delta: \Sigma \times Q \rightarrow Q$ is extended to $\delta: \Sigma^{*} \times Q \rightarrow Q$ in the standard way. Then the language generated by $P$ is defined by

$$
\mathcal{L}(P)=\left\{t \in \Sigma^{*} \mid \delta\left(t, q_{0}\right) \text { is defined }\right\}
$$

The transition alphabet $\Sigma$ consists of two disjoint subsets, ths set $\Sigma_{c}$ of controlled events that can be disabled by external control, and the set $\Sigma_{u}$ of uncontrolled events that cannot be prevented from occurring.

A supervisor $S$ for $P$ is a device that follows the behavior of $P$ and at each state of $P$ disables a subset of the controlled events. The language generated by the (closed-loop) system, i.e., by the combination of $P$ and $S$, is denoted by $\mathcal{L}(S / P)$.

Let $\mathcal{M}$ be a closed sublanguage of $\mathcal{L}(P)$. Then there exists a supervisor $S$ such that $\mathcal{L}(S / P)=\mathcal{M}$ if and only if $\mathcal{M}$ is controllable with respect to $\mathcal{L}(P)$ (see [1]), namely, if and only if

$$
\overline{\mathcal{M}} \Sigma_{u} \bigcap \mathcal{L}(P) \subset \overline{\mathcal{M}}
$$

For any language $\mathcal{M} \subset \mathcal{L}(P)$ there exists a supremal (in the sense of languages inclusion) controllable sublanguage of $\mathcal{M}$, denoted by $\sup C(\mathcal{M})$. Let a specification of legal behavior for $P$ be given as a nonempty sublanguage $\mathcal{E} \subset \Sigma^{*}$. The basic control problem is then to construct a supervisor $S$ such that $\mathcal{L}(S / P) \subset \mathcal{E}$. It is then known (see e.g. [1]) that the optimal solution to this problem is the supervisor $S$ that satisfies the condition that $\mathcal{L}(S / P)=\sup C(\mathcal{L}(P) \cap \mathcal{E})$.

Cieslak et al. in [4] and Lin and Wonham in [5] developed decentralized control schemes, in which instead of a single global supervisor, there are several local supervisors that operate concurrently. Each local supervisor observes and controls only a subset of the events of the system. In [4] a necessary and sufficient condition was introduced which guarantees that the decentralized control scheme achieves the global optimal behavior.

Most practical real-life discrete event systems are concurrent DESs, i.e. they consist of a large number of subsystems that operate concurrently. The event sets of the subsystems may be (pairwise) disjoint, in which case the systems operate concurrently but asynchronously,

\footnotetext{
${ }^{1}$ We omit from the model the subset of marked states, which is not used in the present paper.
} 
or they may partially overlap, yielding a degree of synchronization through the simultaneity of events.

In the present paper we examine the decentralized control of concurrent DESs. We assume that the DES $P$ is composed of concurrent subsystems, $P_{i}$, with event sets $\Sigma_{i}, i=1,2, \ldots, n$. Suppose that for each subsystem $P_{i}$ there is a local supervisor $S_{i}$ that observes and controls only the events in $\Sigma_{i}$. The global controlled system can then be obtained as the concurrent operation of the individually controlled subsystems $S_{i} / P_{i}, \quad 1 \leq i \leq n$.

While the results of [4] and [5] apply to the present situation as well, they have been developed without any assumption on the structure of the DES under consideration. In the present paper we explore some specific properties of decentralized supervisory control that do not apply in the case of unstructured systems.

The paper is organized as follows. In Section 2 we define the key property of separability of languages and describe some properties of separable languages. The formal definition of concurrent DES and the correspondence between a global controllable language and local controllable languages are discussed in Section 3. In Section 4 we formulate the concurrent supervisory control problem and develop two concurrent control schemes under which optimal global behavior can be achieved.

\section{Separable Languages}

Let $\phi \neq \hat{\Sigma} \subset \Sigma$ and define the projection $\pi: \Sigma^{*} \rightarrow \hat{\Sigma}^{*}$ by

$$
\begin{aligned}
& \pi(\epsilon)=\epsilon \\
& \pi(t \sigma)= \begin{cases}\pi(t) \sigma & \text { if } \sigma \in \hat{\Sigma} \\
\pi(t) & \text { otherwise. }\end{cases}
\end{aligned}
$$

The effect of $\pi$ on $t \in \Sigma^{*}$ is to delete from $t$ all symbols not in $\hat{\Sigma}$.

Let $\Sigma_{i}, \quad 1 \leq i \leq n$, be nonempty (not necessarily disjoint) alphabets, and write

$$
\Sigma=\bigcup_{i=1}^{n} \Sigma_{i} .
$$

Define projections $\pi_{i}: \Sigma^{*} \rightarrow \Sigma_{i}^{*}$ as described in (2.1). For a finite set of languages $\left\{\mathcal{L}_{i} \subset\right.$ $\left.\Sigma_{i}^{*}\right\}_{i=1}^{n}$ we define the synchronized product of $\left\{\mathcal{L}_{i}\right\}$, denoted $\|_{i=1}^{n} \mathcal{L}_{i}$, as

$$
\|_{i=1}^{n} \mathcal{L}_{i}=\left\{t \in \Sigma^{*} \mid(\forall i) \pi_{i}(t) \in \mathcal{L}_{i}\right\} .
$$

Clearly, (2.3) is equivalent to the following definition

$$
\|_{i=1}^{n} \mathcal{L}_{i}=\bigcap_{i=1}^{n} \pi_{i}^{-1}\left(\mathcal{L}_{i}\right) .
$$


If $(\forall i \neq j) \Sigma_{i} \cap \Sigma_{j}=\phi$ then $\|_{i=1}^{n} \mathcal{L}_{i}$ is the usual language interleaving, or shuffle product (see [9]) and if $(\forall i, j) \Sigma_{i}=\Sigma_{j}$ then $\|_{i=1}^{n} \mathcal{L}_{i}=\bigcap_{i=1}^{n} \mathcal{L}_{i}$.

Definition 2.1 Let $\Sigma$ be defined as in (2.2) and let $\mathcal{L} \subset \Sigma^{*}$. We shall say that $\mathcal{L}$ is separable with respect to (w.r.t.) $\left\{\Sigma_{i}\right\}_{i=1}^{n}$ if there exists a set of languages $\mathcal{L}_{i} \subset \Sigma_{i}^{*}, \quad 1 \leq i \leq n$, called a generating set of $\mathcal{L}$, such that $\mathcal{L}=\|_{i=1}^{n} \mathcal{L}_{i}$.

\section{Examples}

Let $n=2, \quad \Sigma_{1}=\left\{\alpha, \beta, \gamma_{1}\right\}, \quad \Sigma_{2}=\left\{\alpha, \beta, \gamma_{2}\right\}$. The language $\mathcal{L}=\overline{\gamma_{1} \gamma_{2}+\gamma_{2} \gamma_{1}}$ is separable w.r.t. $\left\{\Sigma_{1}, \Sigma_{2}\right\}$ since $\mathcal{L}=\|_{i=1}^{2} \mathcal{L}_{i}$, where $\mathcal{L}_{1}=\overline{\gamma_{1}}$ and $\mathcal{L}_{2}=\overline{\gamma_{2}}$. Consider now the language $\mathcal{L}=\overline{\gamma_{1} \gamma_{2}}$. Clearly the languages $\left\{\overline{\gamma_{1}}, \overline{\gamma_{2}}\right\}$ are not a generating set of $\mathcal{L}$. It is easy to verify that there do not exist languages $\mathcal{L}_{1}$ and $\mathcal{L}_{2}$ such that $\mathcal{L}=\|_{i=1}^{2} \mathcal{L}_{i}$, thus $\mathcal{L}$ is not separable. Let $\mathcal{L}=\overline{\gamma_{1} \gamma_{2} \alpha+\gamma_{2} \gamma_{1} \alpha}$. It is easy to verify that for $\mathcal{L}_{11}=\overline{\gamma_{1} \alpha}$ and $\mathcal{L}_{12}=\overline{\gamma_{2} \alpha}$ as well as for $\mathcal{L}_{21}=\overline{\gamma_{1} \alpha \beta}$ and $\mathcal{L}_{22}=\overline{\gamma_{2} \alpha \alpha}, L=\|_{i=1}^{2} \mathcal{L}_{j i}$ for $j=1,2$.

As we can see from the last example, for a separable language there may exist several generating sets. For a nonempty separable language $\mathcal{L} \subset \Sigma^{*}$, let $B(\mathcal{L})$ be the set of all generating sets of $\mathcal{L}$, that is,

$$
B(\mathcal{L})=\left\{\left(\mathcal{L}_{1 \alpha}, \mathcal{L}_{2 \alpha}, \ldots, \mathcal{L}_{n \alpha}\right) \mid(\forall \alpha) \mathcal{L}_{i \alpha} \subset \Sigma_{i}^{*} \quad \text { and } \quad \mathcal{L}=\|_{i=1}^{n} \mathcal{L}_{i \alpha}\right\}
$$

The following two properties are automatic

$$
\begin{aligned}
& \|_{i=1}^{n}\left(\bigcup_{\alpha} \mathcal{L}_{i \alpha}\right) \supset \mathcal{L}, \\
& \|_{i=1}^{n}\left(\bigcap_{\alpha} \mathcal{L}_{i \alpha}\right) \subset \mathcal{L} .
\end{aligned}
$$

For each $i, \quad 1 \leq i \leq n$, let $\left\{\mathcal{L}_{i \alpha}\right\}$ be the family of languages consisting of the $\mathrm{i}$-th components of all the elements in $B(\mathcal{L})$. A question that arises is whether the inclusion $(2.7)$ is, in fact, an equality. In other words, whether $B(\mathcal{L})$ contains a generating set $\left(\underline{\mathcal{L}}_{1}, \ldots, \underline{\mathcal{L}}_{n}\right)$ such that each $\underline{\mathcal{L}}_{i}$ is the infimal element (in the sense of language inclusion) of $\left\{\mathcal{L}_{i \alpha}\right\}$. The answer is given in the following proposition.

Proposition 2.1 For each $i, \quad 1 \leq i \leq n$, the nonempty family $\left\{\mathcal{L}_{i \alpha}\right\}$ is closed under arbitrary intersection and

$$
\bigcap_{\alpha} \mathcal{L}_{i \alpha}=\pi_{i}(\mathcal{L})
$$

Corollary 2.1 A language $\mathcal{L} \subset \Sigma^{*}$ is separable w.r.t. $\left\{\Sigma_{i}\right\}_{i=1}^{n}$ if and only if

$$
\mathcal{L}=\|_{i=1}^{n} \pi_{i}(\mathcal{L})
$$


Corollary 2.2 Let $\left(\mathcal{L}_{1}, \ldots, \mathcal{L}_{n}\right) \in B(\mathcal{L})$. Then for each $i$,

$$
\left(\mathcal{L}_{1}, \ldots, \mathcal{L}_{i-1}, \pi_{i}(\mathcal{L}), \mathcal{L}_{i+1}, \ldots, \mathcal{L}_{n}\right) \in B(\mathcal{L})
$$

The last corollary states that if we replace in a generating set $\left(\mathcal{L}_{1}, \ldots, \mathcal{L}_{n}\right)$ of $\mathcal{L}$, any of the components, say $\mathcal{L}_{i_{1}}, \ldots, \mathcal{L}_{i_{k}}$, with the corresponding projections $\pi_{i_{1}}(\mathcal{L}), \ldots, \pi_{i_{k}}(\mathcal{L})$, then the result is also a generating set of $\mathcal{L}$.

The next proposition states that the family $\left\{\mathcal{L}_{i \alpha}\right\}$ is closed also under union.

Proposition 2.2 For each $i, \quad 1 \leq i \leq n$, the nonempty family $\left\{\mathcal{L}_{i \alpha}\right\}$ is closed under arbitrary union, and

$$
\bigcup_{\alpha} \mathcal{L}_{i \alpha}=\pi_{i}(\mathcal{L}) \bigcup\left(\Sigma_{i}^{*}-\pi_{i}\left(\hat{\mathcal{L}}_{i}\right)\right)
$$

where $\hat{\mathcal{L}}_{i}=\bigcap_{\substack{j=1 \\ j \neq i}}^{n} \pi_{j}^{-1}\left(\pi_{j}(\mathcal{L})\right)$.

We conclude this section with the following result.

Proposition 2.3 The set of all separable (w.r.t. $\left\{\Sigma_{i}\right\}_{i=1}^{n}$ ) languages, over $\Sigma$, is closed under arbitrary intersection.

\section{Concurrent Discrete Event Systems}

Interconnected subsystems can be represented by modelling each subsystem as a DES and describing the connections between them in such a way that the combined DES can be obtained in a mechanical way. One such connection is as follows (see e.g. Hoare [10, section 2.3]) Let $P_{i}=\left(Q_{i}, \Sigma_{i}, \delta_{i}, q_{0 i}\right), i=1,2 \ldots, n$, be DESs with control partitions $\Sigma_{i}=\Sigma_{i c} \bigcup \Sigma_{i u}$ such that

$$
(\forall i \neq j) \Sigma_{i u} \bigcap \Sigma_{j}=\phi
$$

This assumption means that there is no synchronization between uncontrolled events of one subsystem with events of another (because we regard uncontrolled events as resulting from spontaneous and unpredictable behavior of the individual subsystems). For each $\sigma \in \Sigma$ let $\operatorname{In}(\sigma)=\left\{i \mid \sigma \in \Sigma_{i}\right\}$. The model of the entire system $P$ is defined by

$$
P=\left(Q, \Sigma, \delta, q_{0}\right)
$$


where $Q=Q_{1} \times Q_{2} \times \cdots \times Q_{n}, \Sigma=\bigcup_{i=1}^{n} \Sigma_{i}\left(\right.$ with $\left.\Sigma_{c}=\bigcup_{i=1}^{n} \Sigma_{i c}, \Sigma_{u}=\bigcup_{i=1}^{n} \Sigma_{i u}\right)$, where $q_{0}=\left(q_{01}, q_{02}, \ldots, q_{0 n}\right)$, and where $\delta: \Sigma \times Q \rightarrow Q$ is given by

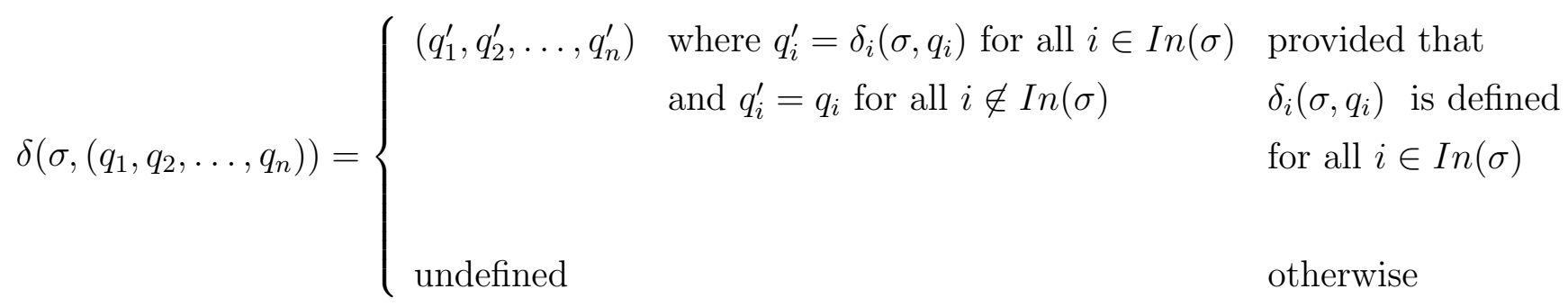

We shall use the notation $P=\|_{i=1}^{n} P_{i}$ for the entire system ${ }^{2}$.

Thus, in the system $P$, an event that belongs to exactly one subsystem can occur asynchronously and independently. But if an event belongs to several subsystems, then it occurs in the composite system if and only if it occurs simultaneously in all the corresponding subsystems. In particular, if the $\Sigma_{i}$ are all disjoint, then $P$ is the shuffle product of $P_{i}$, (see $[1])$.

The next proposition follows immediately from the definition of $P$.

Proposition 3.1 Let $P=\|_{i=1}^{n} P_{i}$, then $\mathcal{L}(P)=\|_{i=1}^{n} \mathcal{L}\left(P_{i}\right)$.

Example 3.1 We consider two machines $P_{1}, P_{2}$ that operate concurrently. The $P_{i}$ are DESs with state diagrams as shown in Fig. 3.1. In state $I_{i}, P_{i}$ is idle and in state $W_{i}$ it is working. Initially the system is in state $\left(I_{1}, I_{2}\right)$. The sets of uncontrolled events are $\Sigma_{1 u}=\left\{b_{1}\right\}, \Sigma_{2 u}=$ $\left\{b_{2}\right\}$, respectively. The system operates as follows. There is an external storage of three types of workpieces for processing. Machine $P_{1}$ takes a workpiece either from the external storage (event $a_{11}$, if the workpiece is of the first type, or event $a_{12}$ if it is of the second type), or from machine $P_{2}$ (shared event $\alpha$ ). If the processing of a workpiece is successfully completed, then $P_{1}$ passes the workpiece to an external storage of processed workpieces (event $b_{1}$ ). Otherwise, it passes the workpiece to machine $P_{2}$ (shared event $\beta$ ). Machine $P_{2}$ operates in the same way, but takes from the external storage only workpieces of the third type (event $\left.a_{2}\right)$. The state diagram of the entire system $P=\|_{i=1}^{2} P_{i}$ is given in Fig. 3.2.

As we mentioned in Section 1, the controllability property plays the key role in supervisor synthesis problems. For concurrent DES $P=\|_{i=1}^{n} P_{i}$ the controllability property has two aspects; global controllability, i.e.; w.r.t. $\mathcal{L}(P)$, and local controllability, i.e.; w.r.t. $\mathcal{L}\left(P_{i}\right)$. More specifically, let $\mathcal{M} \subset \mathcal{L}(P)$ be a "global" sublanguage, the corresponding local

\footnotetext{
${ }^{2}$ We denote by $\|_{i=1}^{n}$ the synchronized product of languages as well as the concurrent composition of systems. The precise meaning will be clear from the context.
} 
languages are $\pi_{i}(\mathcal{M}), \quad 1 \leq i \leq n$. The question now of interest is whether the global controllability of $\mathcal{M}$ implies the local controllability of $\pi_{i}(\mathcal{M})$ or vice versa. (Similar questions appear in [4] for unstructured systems). The answer is given in the Main Lemma.

\section{Lemma 3.1 (Main Lemma).}

Consider a DES $P=\|_{i=1}^{n} P_{i}$.

(a) If $\mathcal{M} \subset \mathcal{L}(P)$ is closed and controllable w.r.t. $\mathcal{L}(P)$, then for each $i, \quad 1 \leq i \leq n$, $\pi_{i}(\mathcal{M}) \subset \mathcal{L}\left(P_{i}\right)$ and $\pi_{i}(\mathcal{M})$ is closed and controllable w.r.t. $\mathcal{L}\left(P_{i}\right)$.

(b) Let $\mathcal{M}_{i} \subset \mathcal{L}\left(P_{i}\right), \quad 1 \leq i \leq n$, be closed and controllable w.r.t. $\mathcal{L}\left(P_{i}\right)$ and write $\mathcal{M}=$ $\|_{i=1}^{n} \mathcal{M}_{i}$. Then $\mathcal{M} \subset \mathcal{L}(P)$ and $\mathcal{M}$ is closed and controllable w.r.t. $\mathcal{L}(P)$.

The Main Lemma motivates all the results about synthesis of supervisors for concurrent DES that we develop in the next Section.

\section{The Supremal Controllable Legal Behavior and Su- pervisor Synthesis}

Consider a DES $P=\|_{i=1}^{n} P_{i}$ as defined in (3.2). Let $\mathcal{E} \subset \Sigma^{*}$ be a nonempty closed language, which we interpret as a specification of legal behavior for $P$. The basic control problem is to synthesize a control device whose task is to restrict the behavior of $P$ to within the specified legal behavior $\mathcal{E}$. As was mentioned in Section 1, a global supervisor $S$ achieves optimal behavior of $P$ by synthesizing the language

$$
\mathcal{K}=\sup C(\mathcal{L}(P) \bigcap \mathcal{E}) \subset \Sigma^{*}
$$

Instead of using a single global supervisor, we propose a decentralized control scheme, called concurrent control. By concurrent control we mean that for each substem $P_{i}$ there is a local supervisor $S_{i}$. Each supervisor $S_{i}$ observes and controls only the events that belong to $\Sigma_{i}$. The concurrent operation of all controlled subsystems $S_{i} / P_{i}$ generates a new global system $P_{c l}$, namely $P_{c l}=\|_{i=1}^{n}\left(S_{i} / P_{i}\right)$. The behavior of $P_{c l}$ is given by

$$
\tilde{\mathcal{K}}:=\mathcal{L}\left(P_{c l}\right)=\|_{i=1}^{n} \mathcal{L}\left(S_{i} / P_{i}\right)=\bigcap_{i=1}^{n} \pi_{i}^{-1}\left(\mathcal{L}\left(S_{i} / P_{i}\right)\right) .
$$

In the remainder of this section, we discuss the relations between the language $\tilde{\mathcal{K}}$, that can be achieved by concurrent control, and the global optimal language $\mathcal{K}$.

The following proposition states the (fairly obvious) fact that concurrent control can never be better than global control. 
Proposition 4.1 Suppose that there exist local supervisors $S_{i}, 1 \leq i \leq n$, such that $\tilde{\mathcal{K}} \subset \mathcal{E}$. Then $\tilde{\mathcal{K}} \subset \mathcal{K}$.

The following theorem gives conditions under which a concurrent control scheme can achieve the optimal global behavior.

Theorem 4.1 Let $P=\|_{i=1}^{n} P_{i}$, where $P_{i}=\left(Q_{i}, \Sigma_{i}, \delta_{i}, q_{0 i}\right), 1 \leq i \leq n$. There exist local supervisors $S_{i}, \quad 1 \leq i \leq n$, such that $\tilde{\mathcal{K}}=\mathcal{K}$ if and only if $\mathcal{K}$ is separable w.r.t. $\left\{\Sigma_{i}\right\}_{i=1}^{n}$.

Remark: In [4] the following decentralized control scheme was examined. Let $P$ be a DES with controlled events $\Sigma_{c}$, where $\Sigma_{c}$ is the union of $n$ subsets $\Sigma_{1 c}, \ldots, \Sigma_{n c}$. Also given are $\mathrm{n}$ event sets $\Delta_{1}, \ldots, \Delta_{n}$ and maps (or masks) $M_{i}: \Sigma \rightarrow \Delta_{i} \bigcup\{\epsilon\}$. A decentralized supervisor is a collection of local supervisors $S_{i}$, where each supervisor $S_{i}$ observes only the symbols belonging to $\Delta_{i}$ and controls only the events of $\Sigma_{i c}$. It was proved in Lemma 4.2 of [4] that $\mathcal{L}\left(\left(\wedge_{i} S_{i}\right) / P\right)=\mathcal{K},\left(\right.$ with $\mathcal{K}$ as defined in (4.1) above), if and only if $\mathcal{K}$ is $\left(\left\{M_{i}\right\},\left\{\Sigma_{i c}\right\}, \mathcal{L}(P)\right)-$ controllable. Namely, for $\sigma \in \Sigma_{c}$, for a set $\left\{s_{i}\right\}$ of strings in $\mathcal{K}$ indexed by $i \in \operatorname{In}(\sigma)$, and for $s^{\prime} \in \mathcal{K}$

(1) $s_{i} \sigma \in \mathcal{K}$ for all $i \in \operatorname{In}(\sigma)$,

(2) $s^{\prime} \sigma \in \mathcal{L}(P)$

(3) $M_{i}\left(s_{i}\right)=M_{i}\left(s^{\prime}\right)$ for all $i \in \operatorname{In}(\sigma)$

together imply

$$
s^{\prime} \sigma \in \mathcal{K} .
$$

It can be shown that if the masks $M_{i}$ are deletion masks, that is, $M_{i}(\sigma) \in\{\sigma, \epsilon\}$ for all $\sigma \in \Sigma$, and if $\Sigma_{i c} \subset \Delta_{i}$ for all $i=1,2, \ldots, n$, then $\mathcal{K}$ is $\left(\left\{M_{i}\right\},\left\{\Sigma_{i c}\right\}, \mathcal{L}(P)\right)$-controllable if and only if

$$
\mathcal{K}=\mathcal{L}(P) \bigcap_{i=1}^{n} \pi_{i}^{-1}\left(\pi_{i}(\mathcal{K})\right)
$$

If, in addition, $P=\|_{i=1}^{n} P_{i}$, where $P_{i}=\left(Q_{i}, \Sigma_{i}, \delta_{i}, q_{0 i}\right)$, and $\Delta_{i} \subset \Sigma_{i}$ for all $i=1,2, \ldots, n$, then $\mathcal{K}$ is $\left(\left\{M_{i}\right\},\left\{\Sigma_{i c}\right\}, \mathcal{L}(P)\right)$-controllable if and only if

$$
\mathcal{K}=\bigcap_{i=1}^{n} \pi_{i}^{-1}\left(\pi_{i}(\mathcal{K})\right) .
$$

Thus under the assumptions of Theorem 4.1, the condition of [4] is equivalent to the separability of $\mathcal{K}$. The separability property can be verified algorithmically. The algorithmic aspects will be discussed later. 
Example 4.1 Consider the system $P=\|_{i=1}^{2} P_{i}$ as defined in example 3.1. Suppose that our aim is to satisfy the following specification. Each processed workpiece of the first type must be passed to the external storage only by $P_{1}$. In other words, if $P_{1}$ passes a workpiece of the first type to $P_{2}$ then $P_{2}$ must return this workpiece to $P_{1}$. Formally, the legal behavior $\mathcal{E}$ is described by the state diagram displayed in Fig. 4.1. In this diagram $\Sigma=\left\{a_{11}, a_{12}, b_{1}, a_{2}, b_{2}, \alpha, \beta\right\}$ and we use also arcs labelled with a set of symbols instead of an individual arc for each symbol. The supremal controllable sublanguage of $\mathcal{L}(P) \bigcap \mathcal{E}, \mathcal{K}$ and its projections on $\Sigma_{1}^{*}$ and $\Sigma_{2}^{*}$ are given in Figs. 4.2 and 4.3, respectively.

It is easily verified that $\mathcal{K}=\|_{i=1}^{2} \pi_{i}(\mathcal{K})$, whence the optimal behavior can be achieved by using only a local supervisor for $P_{1}$ such that $\mathcal{L}\left(S_{1} / P_{1}\right)=\pi_{1}(\mathcal{K})$.

In the case that the language $\mathcal{K} \subset \Sigma^{*}$ is regular it can be determined whether $\mathcal{K}$ is separable w.r.t. subsets $\Sigma_{1}, \Sigma_{2}, \ldots, \Sigma_{n}$. Let $A=\left(X, \Sigma, \xi, x_{0}\right)$ be a deterministic automaton with $m$ states such that $\mathcal{L}(A)=\mathcal{K}$. Clearly the separability of $\mathcal{K}$ can be checked simply by employing the definition of separable languages. The complexity of such an algorithm is $O\left(m^{n+1}\right)$. Clearly, this exponential complexity makes the verification of separability generally infeasible.

Next we introduce an algorithm for checking the separability of a language $\mathcal{K}$ when the subsets $\Sigma_{1}, \ldots, \Sigma_{n}$ are pairwise disjoint. It is easy to see that in this case the separability of a language is equivalent to its being the shuffle product of its projections. We shall see that the algorithm has only polynomial complexity.

First we prove the following motivating lemma.

Lemma 4.1 Let $\mathcal{L} \subset \Sigma^{*}$ be a prefix closed language, and let $\Sigma_{1}, \ldots, \Sigma_{n}$ be pairwise disjoint subsets of $\Sigma$. Then $\mathcal{L}$ is separable w.r.t. $\left\{\Sigma_{i}\right\}_{i=1}^{n}$ if and only if for any $t \in \mathcal{L}$ and $\sigma \in \Sigma_{i}$

$$
\text { to } \notin \mathcal{L} \Longrightarrow(\forall s \in \mathcal{L}) \text { either } \pi_{i}(s) \neq \pi_{i}(t), \quad \text { or } \quad s \sigma \notin \mathcal{L}
$$

We introduce now the algorithm. Again, let $A=\left(X, \Sigma, \xi, x_{0}, X\right)$ be a deterministic automaton that accepts $\mathcal{K}$ and has $m$ states. Let $\Sigma_{1}, \ldots, \Sigma_{n}$ be pairwise disjoint subsets of $\Sigma$.

Algorithm 4.1 (1) For each $i=1,2, \ldots, n$, construct the automaton $A_{i}=\left(X, \Sigma_{i}, \xi_{i}, X_{i 0} X\right)$ as defined in step (2) of Algorithm 4.1.

(2) For each pair $(i, x)$, where $i \in\{1,2, \ldots, n\}$ and $x \in X$, define $A_{i x}=\left(X, \Sigma_{i}, \xi_{i}, X_{i 0}\{x\}\right)$, and define $d_{i}(x)=\left\{\sigma \in \Sigma_{i} \mid \xi_{i}(\sigma, x)=\phi\right\}$. 
(2a) Construct the product automaton $A_{i x} \times A_{i}=\left(X \times X, \Sigma_{i}, \delta, X_{i 0} \times X_{i 0},\{x\} \times X\right)$.

Define $X_{i x} \subset X$ to be the set of all states $x^{\prime} \in X$ such that $\left(x, x^{\prime}\right)$ is an accessible state in $A_{i x} \times A_{i}$, that is

$$
X_{i x}=\left\{x^{\prime} \in X \mid\left(\exists t \in \Sigma_{i}^{*}, \exists x_{10}, x_{20} \in X_{i 0}\right)\left(x, x^{\prime}\right) \in \delta\left(t,\left(x_{10}, x_{20}\right)\right\} .\right.
$$

(2b) If there exists $x^{\prime} \in X_{i x}$ such that $d_{i}\left(x^{\prime}\right) \not \supset d_{i}(x)$, then stop.

(3) If all the pairs $(i, x)$ were checked then stop. Else repeat step (2) for another pair $(i, x)$.

The intuitive concept of the algorithm is as follows. For each $(i, x), \mathcal{L}\left(A_{i x}\right)$ is the set of all strings $\pi_{i}(t)$, such that $t \in \mathcal{K}$ and $\xi\left(t, x_{0}\right)=x$, and $d_{i}(x)$ is the set of all $\sigma \in \Sigma_{i}$ such that $t \sigma \notin \mathcal{K}$. By constructing $A_{i x} \times A_{i}$ the algorithm identifies the set $X_{i x}$ which is the set of all states $x^{\prime} \in X$ such that there exist $s, t \in \mathcal{K}$ which satisfies that $\pi_{i}(s)=\pi_{i}(t)$ and that $\xi\left(t, x_{0}\right)=x$ and $\xi\left(s, x_{0}\right)=x^{\prime}$. Now if $d_{i}\left(x^{\prime}\right) \not \supset d_{i}(x)$ then there exists $\sigma \in \Sigma$ such that $t \sigma \notin \mathcal{K}$ and $s \sigma \in \mathcal{K}$ which contradicts the condition of Lemma 4.1.

The correctness of this algorithm is formally stated in the following proposition.

Proposition $4.2 \mathcal{K}$ is separable w.r.t. $\left\{\Sigma_{i}\right\}_{i=1}^{n}$ if and only if Algorithm 4.2 stops at step (3).

The complexity of algorithm 4.2 is $O\left(n \cdot m^{3}\right)$.

In the system $P_{c l}$ there are two mechanisms that restrict the behavior of the subsystems $P_{i}$. One is the control mechanism, i.e.; the local supervisor $S_{i}$ prevents the occurrence of some events in $P_{i}$. The second is the synchronization mechanism that prevents a shared event $\sigma \in \Sigma_{i}$ from occurring in $P_{i}$ if $\sigma$ cannot occur simultaneously in all subsystems $P_{j}$ that satisfy $\sigma \in \Sigma_{j}$. These mechanisms can overlap in the sense that an event $\sigma \in \Sigma_{i}$ can be prevented from occurring, in some local state of $P_{i}$, by both mechanisms.

The possibility of overlapping disabling mechanisms leads us to think about two possible control strategies. The first is, the most restrictive control, in which the behaviors of the subsystems are restricted by the supervisors as much as possible. In this case the effect of the synchronization mechanism on the subsystems behavior is minimal. The second is, the least restrictive control, in which the effect of the synchronization mechanism is maximal and the supervisors disable an event only if it is not already prevented from occuring by the synchronization mechanism. To formalize this issue, let $C(\mathcal{K})$ be a class of all sets of supervisors that by acting concurrently synthesize the language $\mathcal{K}$,

$$
C(\mathcal{K})=\left\{\left\{S_{1 \alpha}, \ldots, S_{n \alpha}\right\} \mid(\forall \alpha) \quad \mathcal{L}\left(\|_{i=1}^{n}\left(S_{i \alpha} / P_{i}\right)\right)=\mathcal{K}\right\}
$$

The following theorem addresses the issue of the most restrictive control. 
Theorem 4.2 If $\mathcal{K}$ is separable w.r.t. $\left\{\Sigma_{i}\right\}_{i=1}^{n}$, then there exists a set of supervisors $\left\{\overline{S_{i}}, \ldots, \overline{S_{n}}\right\} \in$ $C(\mathcal{K})$ such that for all $\left\{S_{1}, \ldots, S_{n}\right\} \in C(\mathcal{K})$,

$$
\mathcal{L}\left(\overline{S_{i}} / P_{i}\right)=\pi_{i}(\mathcal{K}) \subset \mathcal{L}\left(S_{i} / P_{i}\right), \quad i=1, \ldots, n
$$

There does not, in general, exist a least restrictive control law. To see this, let us examine the following example.

Example 4.2 Consider the systems $P_{1}, P_{2}, P$ and the language $\mathcal{K}$ as shown in Fig. 4.4. Let $\left(S_{11}, S_{12}\right)$ and $\left(S_{21}, S_{22}\right)$ be pairs of supervisors such that

$$
\mathcal{L}\left(S_{11} / P_{1}\right)=\overline{a_{1}}, \quad \mathcal{L}\left(S_{12} / P_{2}\right)=\mathcal{L}\left(P_{2}\right), \quad \mathcal{L}\left(S_{21} / P_{1}\right)=C L\left(P_{1}\right) \quad \text { and } \mathcal{L}\left(S_{22} / P_{2}\right)=\overline{a_{2}} .
$$

Clearly, for $j=1,2, \mathcal{L}\left(\|_{i=1}^{2}\left(S_{j i} / P_{i}\right)\right)=\mathcal{K}$. Thus there does not exit a pair $\left(S_{1}, S_{2}\right)$ that synthesizes the language $\mathcal{K}$ and that $S_{1}$ and $S_{2}$ are the least restrictive supervisors for $P_{1}$ and $P_{2}$, respectively.

We wish to show that if the supervisors $S_{i}$ are synthesized according to some order, then there exists a least restrictive control law. Let $I=\{1,2, \ldots, n\}$ and let $\prec$ be an order on the elements of $I$. We denote by $i_{1}, \ldots, i_{n}$ the elements of $I$ that are ordered according to $\prec$ i.e.; $i_{j} \prec i_{j+1}$ for $j=1,2, \ldots, n-1$. Suppose that we synthesize the supervisors $S_{i}, 1 \leq i \leq n$, according to $\prec$, namely the first supervisor is $S_{i_{1}}$, the second is $S_{i_{2}}$ and so on. In practical applicatins the order $\prec$ can frequently be determined according to the ability to implement the control law. That is, for $i, j \in I, i \prec j$ if it is more difficult or less desirable to implement the disablement of events in $P_{i}$ than in $P_{j}$.

The question now of interest is whether for each $j$ we can synthesize a least restrictive supervisor $S_{i_{j}}$ under the condition that the previous supervisors $S_{i_{1}}, \ldots, S_{i_{j-1}}$ are already given. In other words, does there exist a set of supervisors $\left\{\overline{S_{i_{1}}}, \ldots, \overline{S_{i_{n}}}\right\} \in C(\mathcal{K})$ such that $\overline{S_{i_{1}}}$ is the least restrictive supervisor for $P_{i_{1}}, \overline{S_{i_{2}}}$ is the least restrictive supervisor for $P_{i_{2}}$ under the condition that $\overline{S_{i_{1}}}$ is given and so on. For simplicity we assume below, without loss of generality, that $i_{j}=j$ for $j=1, \ldots, n$.

Suppose that $\mathcal{K} \subset \mathcal{L}(P)$ is a nonempty separable language (w.r.t. $\left\{\Sigma_{i}\right\}_{i=1}^{n}$ ) and denote by $B P(\mathcal{K})$ the set of all generating sets of $\mathcal{K}$ where each component is a closed sublanguage of the corresponding subsystem, that is,

$$
B P(\mathcal{K})=\left\{\left(\mathcal{L}_{1 \alpha}, \ldots, \mathcal{L}_{n \alpha}\right) \mid(\forall \alpha) \mathcal{L}_{i \alpha} \subset \mathcal{L}\left(P_{i}\right), \mathcal{L}_{i \alpha} \text { is closed and } K=\bigcap_{i=1}^{n} \pi_{i}^{-1}\left(\mathcal{L}_{i \alpha}\right)\right\}
$$

We define, inductively, a sequence of closed languages $\mathcal{K}_{i} \subset \mathcal{L}\left(P_{i}\right), \quad i=1,2, \ldots, n$, as follows:

$$
\mathcal{K}_{1}=\bigcup_{\alpha} \mathcal{L}_{1 \alpha}
$$


Let $\left(\mathcal{K}_{1}, \ldots, \mathcal{K}_{l-1}\right)$ be given and denote by $B P_{l}\left(\mathcal{K}_{1}, \ldots, \mathcal{K}_{l-1}\right) \subset B P(\mathcal{K})$ the collection of all generating sets of $\mathcal{K}$ in which the first $l-1$ components are $\mathcal{K}_{1}, \ldots, \mathcal{K}_{l-1}$. That is,

$B P_{l}\left(\mathcal{K}_{1}, \ldots, \mathcal{K}_{l-1}\right)=\left\{\left(\mathcal{K}_{1}, \ldots, \mathcal{K}_{l-1} \mathcal{L}_{l \beta}, \ldots, \mathcal{L}_{n \beta}\right) \mid(\forall \beta)\left(\mathcal{K}_{1}, \ldots, \mathcal{K}_{l-1}, \mathcal{L}_{l \beta}, \ldots, \mathcal{L}_{n \beta}\right) \in B P(\mathcal{K})\right\}$

Clearly $B P_{1}=B P(\mathcal{K})$. The $l$-th language is defined by

$$
\mathcal{K}_{l}=\bigcup_{\beta} \mathcal{L}_{l \beta}
$$

The properties of the sequence $\left(\mathcal{K}_{1}, \ldots, \mathcal{K}_{n}\right)$ are given in the following two lemmas the first of which states that the above construction is sound, i.e.,

\section{Lemma 4.2}

$$
\left(\mathcal{K}_{1}, \ldots, \mathcal{K}_{n}\right) \in B P(\mathcal{K})
$$

The meaning of the last lemma is as follows. If $\mathcal{K}$ is separable, then among all the generating sets of $\mathcal{K}$ for which the components that correspond to the first $j$ subsystems are fixed, there is a set in which the component corresponding to the $j+1$ subsystem is maximal.

Lemma 4.3 For each $j, 1 \leq j \leq n$, the language $\mathcal{K}_{j}$ is controllable w.r.t. $\mathcal{L}\left(P_{j}\right)$.

The last two lemmas motivate the following result.

Theorem 4.3 If $\mathcal{K}$ is separable w.r.t. $\left\{\Sigma_{i}\right\}_{i=1}^{n}$, then there exists a set of supervisors $\left\{\overline{S_{1}}, \ldots, \overline{S_{n}}\right\} \in$ $C(\mathcal{K})$ such that for each $l, l=1,2, \ldots, n$, the following property holds. If $\left\{S_{1}, \ldots, S_{l}, \ldots, S_{n}\right\} \in$ $C(\mathcal{K})$ such that for all $j<l, \mathcal{L}\left(S_{j} / P_{j}\right)=\mathcal{L}\left(\overline{S_{j}} / P_{j}\right)$, then

$$
\mathcal{L}\left(S_{l} / P_{l}\right) \subset \mathcal{L}\left(\overline{S_{l}} / P_{l}\right)=\mathcal{K}_{l}
$$

We present now an algorithm to compute the languages $\mathcal{K}_{j}$. Let

$$
\hat{\mathcal{K}}_{j}=\left[\bigcap_{l=1}^{j-1} \pi_{l}^{-1}\left(\mathcal{K}_{l}\right)\right] \bigcap\left[\bigcap_{l=j+1}^{n} \pi_{l}^{-1}\left(\pi_{l}(\mathcal{K})\right)\right], \quad j=1,2, \ldots, n .
$$

The following result states that for given languages $\mathcal{K}_{1}, \ldots, \mathcal{K}_{j-1}$, the supremal element $\mathcal{K}_{j}$

can be computed as follows. First construct $\hat{\mathcal{K}}_{j}$ the synchronized product of $\mathcal{K}_{1}, \ldots, \mathcal{K}_{j-1}$ and of the infimal elements $\pi_{j+1}(\mathcal{K}), \ldots, \pi_{n}(\mathcal{K})$. Then compute the supremal sublanguage $\mathcal{L}_{j, \text { max }}$ of $\mathcal{L}\left(P_{j}\right)$, such that the synchronized product of $\hat{\mathcal{K}}_{j}$ and $\mathcal{L}_{j, \text { max }}$ yields $\mathcal{K}$.

\section{Proposition 4.3}

$$
\mathcal{K}_{j}=\pi_{j}(\mathcal{K}) \bigcup\left[\mathcal{L}\left(P_{j}\right)-\pi_{j}\left(\hat{\mathcal{K}}_{j}\right)\right], \quad j=1,2, \ldots, n
$$


Example 4.3 Let $P=\|_{i=1}^{2} P_{i}$ be the system described in Example 3.1. When $P_{1}$ and $P_{2}$ operate concurrently, there is a possibility that one subsystem, say $P_{1}$, is an "exploiter". Namely, $P_{1}$ does not process any workpiece, it passes all the workpieces to $P_{2}$. Clearly this is an undesirable situation. Suppose that we require that the passing of workpieces between the subsystems be fair. In particular, it may be required that if $P_{i}$ passes a workpiece to $P_{j}$ then it will not do so again until $P_{j}$ passes a workpiece to $P_{i}$. Thus, a string $t$ of the closed loop behavior is legal only if its projection on $\{\alpha+\beta\}^{*}$ belongs to $(\alpha \beta)^{*}+(\beta \alpha)^{*}$. The legal behavior $\mathcal{E}$ is described by the generator displayed in Fig. 4.5. The optimal language $\mathcal{K}$ and the projections of $\mathcal{K}$ on $\Sigma_{1}^{*}, \Sigma_{2}^{*}$ are described in Figs. 4.6 and 4.7 , respectively. It can be verified that $\mathcal{K}=\|_{i=1}^{2} \pi_{i}(\mathcal{K})$, so $\mathcal{K}$ is separable w.r.t. $\left(\Sigma_{1}, \Sigma_{2}\right)$.

\section{Most Restrictive Control}

¿From Theorem 4.2 it follows that the most restrictive control can be implemented by supervisors $S_{1}, S_{2}$ such that $\mathcal{L}\left(S_{i} / P_{i}\right)=\pi_{i}(\mathcal{K})$.

\section{Least Restrictive Control}

Case (i): $1 \prec 2$

In this case $i_{1}=1$ and $i_{2}=2$, that is, first we synthesize the least restrictive supervisor $S_{1}$ and afterwards the least restrictive supervisor $S_{2}$ under the assumption that $S_{1}$ is given. To construct $S_{i}$ we use Proposition 4.4.

$$
\begin{aligned}
& \hat{\mathcal{K}}_{1}=\pi_{2}^{-1}\left(\pi_{2}(\mathcal{K})\right) \\
& \mathcal{K}_{1}=\pi_{1}(\mathcal{K}) \bigcup\left[\mathcal{L}\left(P_{1}\right)-\pi_{1}\left(\pi_{2}^{-1}\left(\pi_{2}(\mathcal{K})\right)\right)\right] .
\end{aligned}
$$

It can be verified that $\pi_{1}\left(\pi_{2}^{-1}\left(\pi_{2}(\mathcal{K})\right)\right) \bigcap \mathcal{L}\left(P_{1}\right)=\pi_{1}(\mathcal{K})$, thus

$$
\mathcal{K}_{1}=\mathcal{L}\left(P_{1}\right)
$$

Now we compute $\mathcal{K}_{2}$.

$$
\begin{aligned}
\hat{\mathcal{K}}_{2} & =\pi_{1}^{-1}\left(\mathcal{L}\left(P_{1}\right)\right) \\
\mathcal{K}_{2} & =\pi_{2}(\mathcal{K}) \bigcup\left[\mathcal{L}\left(P_{2}\right)-\pi_{2}\left(\pi_{1}^{-1}\left(\mathcal{L}\left(P_{1}\right)\right)\right)\right] .
\end{aligned}
$$

It is easy to verify that $\pi_{2}\left(\pi_{1}^{-1}\left(\mathcal{L}\left(P_{1}\right)\right)\right)=\Sigma_{2}^{*}$, thus

$$
\mathcal{K}_{2}=\pi_{2}(\mathcal{K})
$$

Hence the least restrictive control under the assumption that $1 \prec 2$ is the supervisor $S_{2}$ such that

$$
\mathcal{L}\left(S_{2} / P_{2}\right)=\pi_{2}(\mathcal{K})
$$

Case (ii): $2 \prec 1$ 
By using the same arguments as in the previous case, it can be shown that the least restrictive control under the assumption that $2 \prec 1$ is the supervisor $S_{1}$ such that

$$
\mathcal{L}\left(S_{1} / P_{1}\right)=\pi_{1}(\mathcal{K})
$$

All the results up to this point are concerned with the language $\mathcal{K}$. To compute $\mathcal{K}$ it is necessary to compute the global behavior of the system, $\mathcal{L}(P)$. It is well known that the number of states of $P$, where $P=\|_{i=1}^{n} P_{i}$ increases exponentially with $n$, the number of subsystems. This fact often makes the computation of $\mathcal{L}(P)$ infeasible. Therefore, it is important to find conditions which ensure that the equality $\tilde{\mathcal{K}}=\mathcal{K}$ holds and which can be verified without the computation of $\mathcal{L}(P)$. Such conditions are given in the following theorem.

Theorem 4.4 Consider a DES $P=\|_{i=1}^{n} P_{i}$, where $P_{i}=\left(Q_{i}, \Sigma_{i}, \delta_{i}, q_{0 i}\right), 1 \leq i \leq n$. Suppose that $\mathcal{E} \subset \Sigma^{*}$ is separable w.r.t. $\left\{\Sigma_{i}\right\}_{i=1}^{n}$ and let $\left(\mathcal{E}_{1}, \ldots, \mathcal{E}_{n}\right)$ be a generating set of $\mathcal{E}$, where $\mathcal{E}_{i}$ are closed languages. Let $\mathcal{K}_{i}=\sup C_{i}\left(\mathcal{L}\left(P_{i}\right) \bigcap \mathcal{E}_{i}\right)$, where $\sup C_{i}(\cdot)$ denotes the supremal controllable sublanguage w.r.t. $\mathcal{L}\left(P_{i}\right)$. Then there exist supervisors $S_{i}, 1 \leq i \leq n$, such that

$$
\begin{aligned}
\mathcal{L}\left(S_{i} / P_{i}\right) & =\mathcal{K}_{i}, \quad 1 \leq i \leq n, \quad \text { and } \\
\tilde{\mathcal{K}} & =\mathcal{K} .
\end{aligned}
$$

If the legal behavior $\mathcal{E}$ is given and is separable, then to synthesize the local supervisors it is necessary to compute the language $\pi_{i}(\mathcal{E})$ and $\mathcal{K}_{i}=\sup C_{i}\left(\mathcal{L}\left(P_{i}\right) \bigcap \pi_{i}(\mathcal{E})\right), i=1,2, \ldots, n$. So the computation of $\mathcal{L}(P)$ is not needed.

So far, we assumed that the desired legal behavior of the DES is represented by the language $\mathcal{E}$ within which, the language of the controlled system must reside. In some cases, however, the desired legal behavior is specified by $n$ individual requirements each of which constitutes a restriction on the sequences in $\mathcal{L}\left(P_{i}\right), 1 \leq i \leq n$. In other words, the i-th requirement specifies the desired legal behavior of $P_{i}$. Let $\mathcal{E}_{i} \subset \Sigma_{i}^{*}$ be the set of all strings in $\Sigma_{i}^{*}$ that satisfy the $\mathrm{i}$-th requirement, we assume that $\mathcal{E}_{i}$ are closed languages. Suppose further that the desired global legal behavior is given by

$$
\mathcal{E}=\bigcap_{i=1}^{n} \pi_{i}^{-1}\left(\mathcal{E}_{i}\right) .
$$

Since $\left(\mathcal{E}_{1}, \ldots, \mathcal{E}_{n}\right)$ is a generating set of $\mathcal{E}$, then by using Theorem 4.4 we get the following result.

Theorem 4.5 Consider a DES $P=\|_{i=1}^{n} P_{i}$ and let $\mathcal{E}$ be as defined in (4.6). Then there exist supervisors $S_{i}, 1 \leq i \leq n$, such that $\mathcal{L}\left(S_{i} / P_{i}\right)=\sup C_{i}\left(\mathcal{L}\left(P_{i}\right) \bigcap \mathcal{E}_{i}\right)$ and $\tilde{\mathcal{K}}=\mathcal{K}$.

The last theorem states that if the constraints are given locally, then the synthesis procedure, of supervisors that achieve the optimal behavior, is based only on local information. 


\section{Conclusion}

A specific version of decentralized supervisory control for concurrent discrete-event systems has been introduced. A necessary and sufficient condition and another sufficient condition (which can be verified by using only local information) were obtained which guarantee that the decentralized control is equivalent to global control.

The special aspects of the concept of least restrictive supervision in concurrent systems were also discussed.

\section{References}

[1 ] Ramadge P.J. and Wonham W.M., "Supervisory control of a class of discrete event processes", SIAM J. on Control and Optimization, 25(1), pp. 206-230, January 1987.

[2 ] Ramadge P.J. and Wonham W.M., "Modular feedback logic for discrete even systems", SIAM J. on Control and Optimization, 25(5), pp. 1202-1218, September 1987.

[3 ] Wonham W.M. and Ramadge P.J., "On the supremal controllable sublanguage of a given language", SIAM J. on Control and Optimization, 25(3), pp. 637-659, May 1987.

[4 ] Cieslak C., Declaux C., Fawaz A. and Varaiya P., "Supervisory control of discrete event systems with partial observations", IEEE Trans. Automatic Control, Vol. AC-33, pp. 249-260, March 1988.

[5 ] Lin F. and Wonham W.M., "Decentralized supervisory control of discrete event systems", Information Sciences, 44, pp. 199-224, 1988.

[6 ] Lu W. and Chen Y., "Structuralized control logic for discrete event system", Proc. of the International Conference on Control and Application, Jerusalem, Israel, 1989.

[7 ] Kuratowski K., Topology, Academic Press, New York, 1966.

[8 ] Tadmor G. and Maimon O., "Control of large discrete event systems: constructive algorithms", IEEE Trans. Automatic Control, Vol. AC-34, pp. 1164-1168, November 1989.

[9] Eilenberg S., Automata, languages and machines, Volume A, Academic Press, New York, 1974.

[10 ] Hoare C.A.R., Communicating sequential processes, Prentice Hall, Englewood Cliffs, 1985.

[11 ] Hopcroff J.E. and Ullman J.D., Introduction to Automata Theory Languages and Computation, Addison-Wesley Pub. Co. Reading, 1979. 